\title{
Reverse takotsubo cardiomyopathy presenting as abdominal pain: An atypical presentation
}

\author{
Guramrinder Singh Thind, Yashwant Agrawal \\ Department of Internal Medicine, Western Michigan University School of Medicine, Kalamazoo, United States
}

Received: May 17, 2017

Accepted: August 22, 2017

Online Published: August 28, 2017

DOI: $10.5430 /$ crim.v4n4p1

URL: https://doi.org/10.5430/crim.v4n4p1

\begin{abstract}
Reverse takotsubo cardiomyopathy is the rarest variant of takotsubo cardiomyopathy. We present a case of reverse takotsubo cardiomyopathy presenting as abdominal pain likely due to non-occlusive mesenteric ischemia. A 58-year-old female presented with abdominal pain, nausea, vomiting, and diarrhea for 2 days. Upon presentation, she was normotensive but tachycardic. Her lactic acid level was $2.8 \mathrm{mmol} / \mathrm{L}$ and troponin-T was $0.62 \mathrm{ng} / \mathrm{ml}$. The electrocardiogram showed non-specific ST changes. $\mathrm{CT}$ scan of abdomen revealed bowel wall thickening in transverse colon and splenic flexure. Patient was started on aggressive intravenous fluid therapy for possible infectious colitis.

The next day, patient started becoming progressively more dyspneic, hypoxic, and hypotensive. A chest X-ray at that time showed signs of severe congestive heart failure. Echocardiogram revealed left ventricular ejection fraction of $20 \%$ with akinesis of proximal and mid-ventricular walls. Patient was intubated for hypoxic respiratory failure and started on milrinone infusion for acutely depressed cardiac function. Inotropes were weaned off the next day, and she was successfully extubated on day 4. Subsequently, gastrointestinal symptoms resolved. Cardiac catheterization was performed later during the hospitalization that showed normal coronary arteries. Repeat echocardiogram done 3 months later showed return of cardiac function to normal.
\end{abstract}

Key Words: Reverse takotsubo cardiomyopathy, Non-occlusive mesenteric ischemia, Cardiogenic pulmonary edema, Acute heart failure

\section{INTRODUCTION}

Takotsubo (stress) cardiomyopathy is a recently identified form of non-ischemic cardiomyopathy. In its classical form, it is presents as apical akinesis. Other variants have since been described including reverse (inverted) takotsubo cardiomyopathy, where basal parts of the left ventricle are akinetic. Awareness of the presence of these variants is helpful in diagnostication and prognostication of new-onset cardiomyopathy.

Non-occlusive mesenteric ischemia occurs due to global mesenteric hypoperfusion. It can be easily recognized by its predilection for the watershed areas of gut. Low cardiac output state is an important risk factor for non-occlusive mesenteric ischemia.

\section{Case presentation}

A 58-year-old female presented with abdominal pain, nausea, vomiting, and diarrhea for 3 days. She had a past medical history of chronic obstructive pulmonary disease (COPD), gastroesophageal reflux disease, and degenerative disc disease. She had 20 pack year history of smoking and had quit

\footnotetext{
* Correspondence: Guramrinder Singh Thind; Email: guramrinder.thind@med.wmich.edu; Address: Department of Internal Medicine, Western Michigan University School of Medicine, Kalamazoo, MI 49008, United States.
} 
20 years ago. Her COPD was well controlled with inhaled fluticasone and salmeterol, and she was not on supplemental oxygen at home. She first started noticing abdominal pain 2 days prior to presentation. She noted that the pain was slightly worse after meals. This was accompanied by nausea and vomiting. Patient also had three loose stools the day before the presentation. She reported that the intensity of pain had increased over the last 24 hours, which made her come in to the hospital.

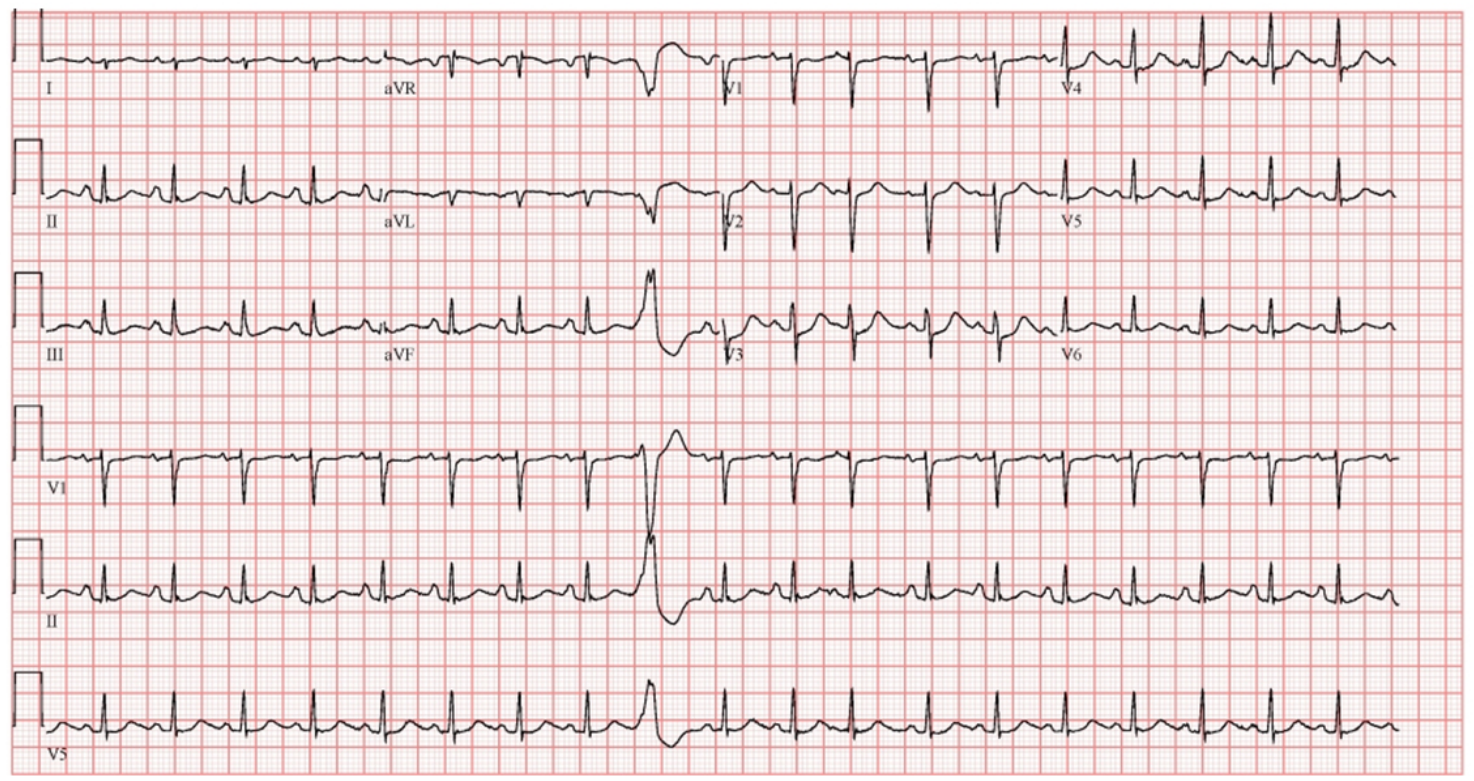

Figure 1. The 12-lead electrocardiogram performed on admission showing ST depression in the anterior leads

The initial set of vitals recorded were: temperature: $98.6^{\circ} \mathrm{F}$, blood pressure: $138 / 92 \mathrm{mmHg}$, heart rate: $128 / \mathrm{min}$, respiratory rate: $16 / \mathrm{min}$, oxygen saturation: $96 \%$ on room air. Her physical exam was unremarkable except for minimal tenderness in the epigastric region. Complete blood count and comprehensive metabolic panel were ordered. She was found to have a white blood cell count of $18,700 / \mu 1$ with
$88 \%$ neutrophils. Her electrolytes and renal function tests were normal. Her lactic acid level was $2.8 \mathrm{mmol} / \mathrm{L}$. Serum troponin-T was checked and was found to be elevated at $0.62 \mathrm{ng} / \mathrm{ml}$. She did not complain of any chest pain. Her electrocardiogram (EKG) showed ST depressions in V3 and V4 leads (see Figure 1).

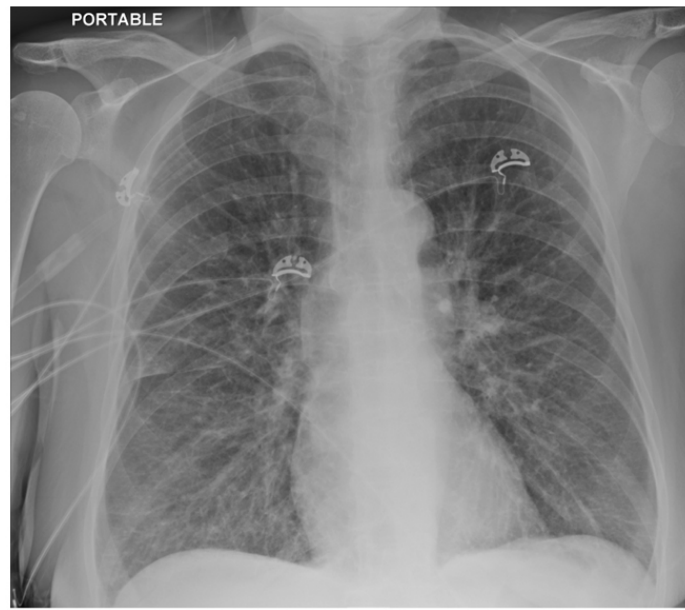

A

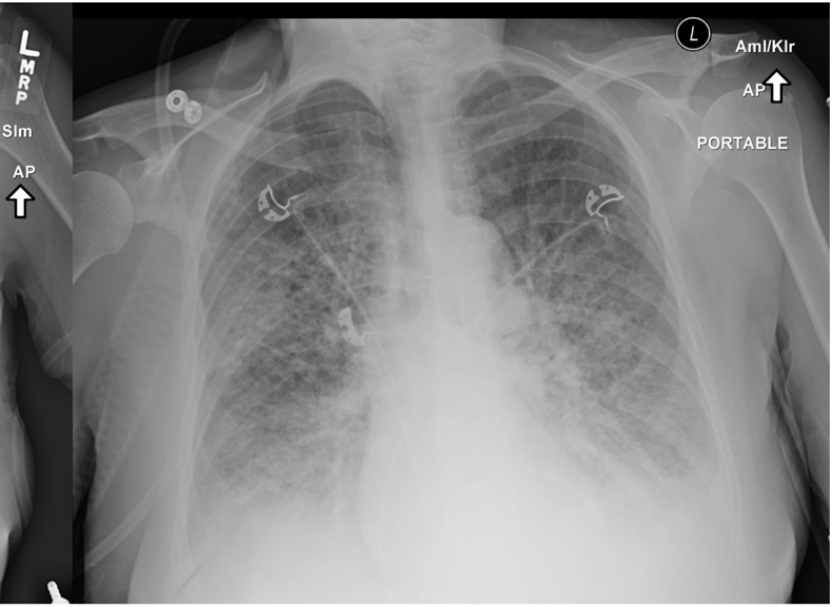

B

Figure 2. Comparison of chest X-ray on admission and after aggressive fluid therapy A) Chest X-ray on admission showing redistribution of blood flow and interstitial pulmonary edema. B) Chest X-ray after fluid therapy showing severe alveolar edema and bilateral pleural effusions. 
Due to persistent severe abdominal pain, patient underwent Computed Tomography (CT) scan of the abdomen with intravenous contrast, which showed bowel wall thickening in the transverse colon and splenic flexure with mild adjacent pericolonic edema. These findings were consistent with radiological evidence of colitis in the transverse colon and splenic flexure. As a side-note, the CT scan also showed reflux of contrast into the hepatic veins, which is typically seen with right heart failure. Due to the tachycardia and elevated lactic acid level, the etiology of patient's colitis was thought to be infectious and she was started on ceftriaxone and metronidazole. At the same time, normal saline boluses were ordered.

Over the next 4-5 hours, patient started reporting shortness of breath. Her oxygen saturation started to drop and she had to be put on 4 liters/minute of supplemental oxygen via nasal cannula. An erect chest X-ray showed redistribution of blood flow in the nondependent portions of the lungs, as well as evidence of interstitial pulmonary edema (kerley A and B lines). However, there was no evidence of alveolar pulmonary edema (see Figure 2). At this time, the patient was admitted to the general floor. Patient received a total of 4 liters of crystalloids in the emergency room. Upon admission, she was started on maintenance fluids at the rate of $200 \mathrm{ml} /$ hour. Repeat lactic acid level later that day was $4.2 \mathrm{mmol} / \mathrm{L}$, and repeat troponin-T was $0.49 \mathrm{ng} / \mathrm{ml}$. When patient was evaluated the next morning, she remained on 4 liters/min of oxygen support. Morning lactic acid level was $4.0 \mathrm{mmol} / \mathrm{L}$. Another 2 liters of normal saline bolus and $500 \mathrm{cc}$ of $5 \%$ albumin bolus were ordered. Over the next 4-5 hours, her respiratory distress worsened and her oxygen requirements increased.

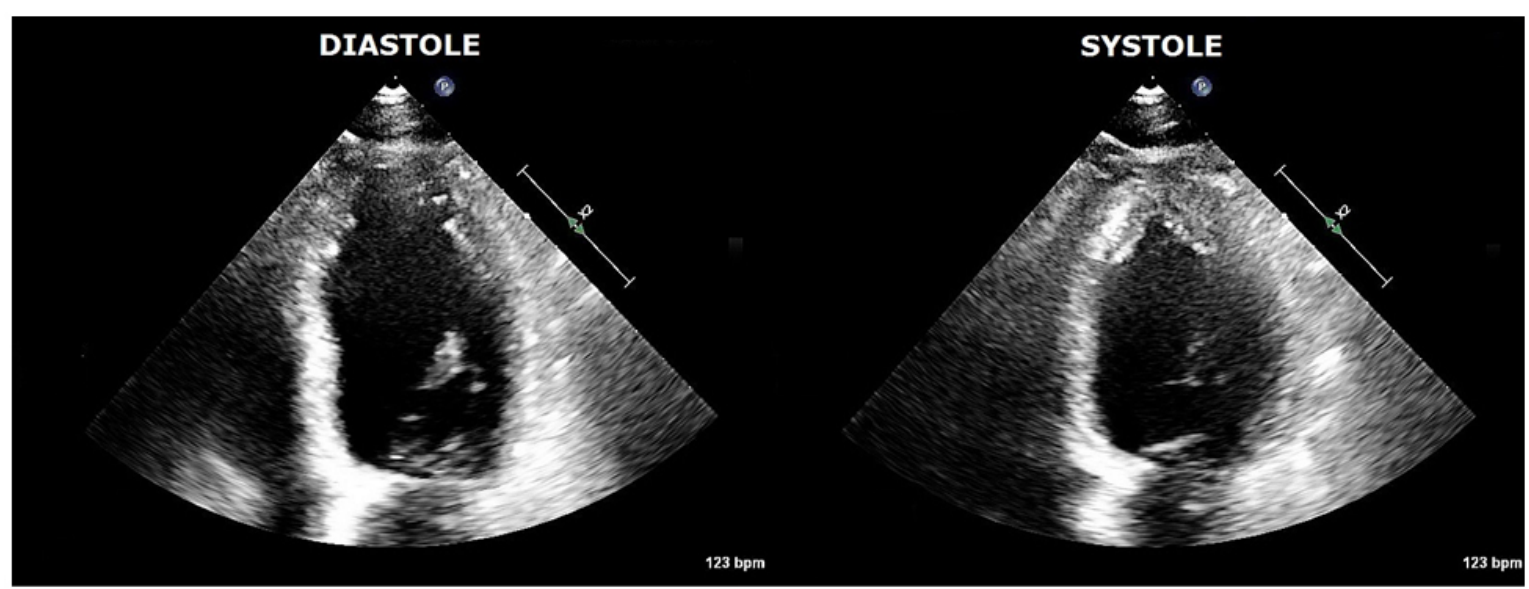

A

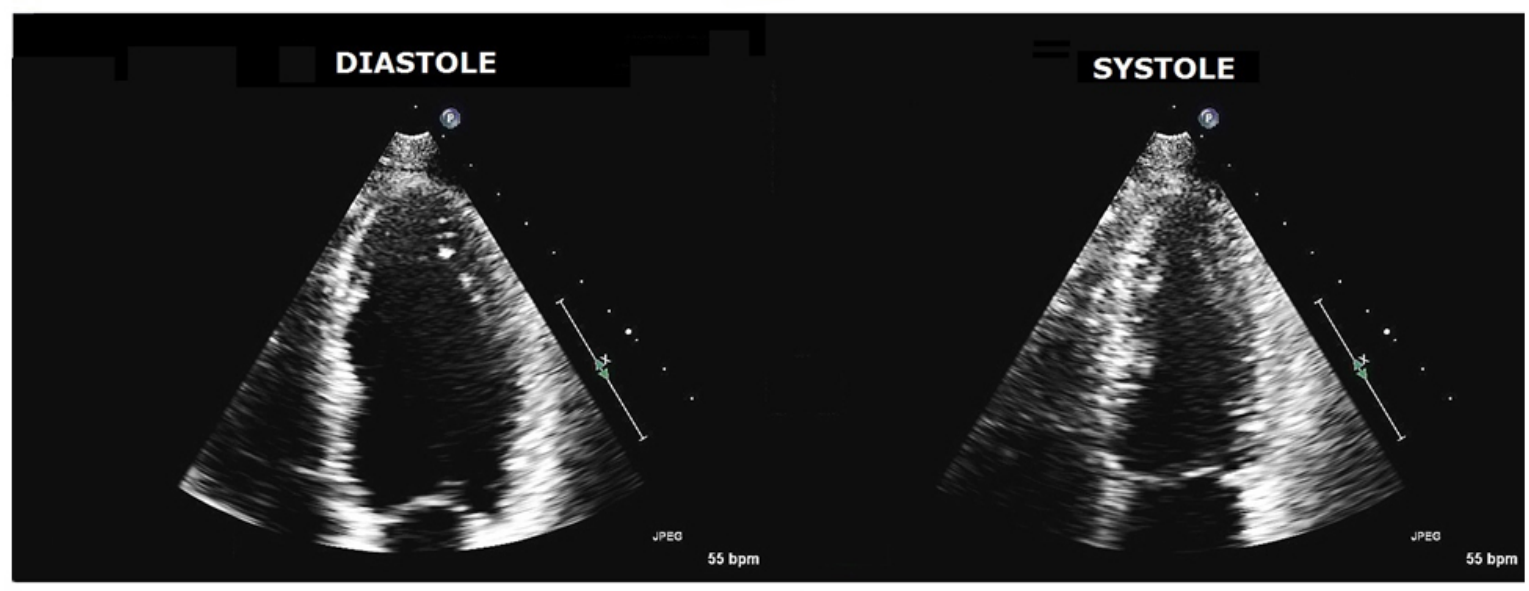

B

Figure 3. Echocardiographic apical four chamber views of the heart focussing on the left ventricle

A) Echocardiogram done during admission showing severely reduced left ventricular systolic function with a visually estimated ejection fraction of 20\%-25\%. The apex is normokinetic but proximal and mid-ventricular walls are akinetic with some basal ballooning noted. B) Echocardiogram done after three months shows resolution of these wall motion abnormalities. 
Thereafter, a repeat chest X-ray was performed that showed significant perihilar alveolar pulmonary edema (see Figure 2 ). She eventually had to be intubated for acute hypoxic respiratory failure and was transferred to the intensive care unit (ICU). In the ICU, a transthoracic echocardiogram was done that showed severely reduced left ventricular systolic function with a visually estimated ejection fraction of $20 \%$ $25 \%$. Intriguingly, the apex was normokinetic but proximal and mid-ventricular walls were akinetic (see Figure 3). There was trace mitral valvular regurgitation. Also, right ventricular size was mildly enlarged and right ventricular systolic function was moderately reduced. The peculiar wall motion abnormalities seen on the echocardiogram were highly suspicious for reverse takotsubo cardiomyopathy. Ischemic cardiomyopathy was on the differential but was less likely. On obtaining further history from family, it was learned that the patient had been very stressed over the past few days due to illness of her daughter.

FloTrac ${ }^{\circledR}$ device was used for precise monitoring of patient's hemodynamics. Patient was initially found to have a depressed cardiac index of $1.9 \mathrm{~L} / \mathrm{min} / \mathrm{m}^{2}$ and was started on milrinone infusion. Troponin levels trended down eventually. Inotropes were weaned off the next day, and she was successfully extubated on day 4. By this time, her gastrointestinal symptoms had resolved. The gastrointestinal polymerase chain reaction (PCR) panel that tests for 21 different gastrointestinal pathogens was negative. Therefore, patient's colitis was determined to be most likely ischemic in nature. Subsequently, cardiac catheterization was performed that showed normal coronary arteries. Her colitis was thought to be due to nonocclusive mesenteric ischemia secondary to severe left ventricular dysfunction in the setting of reverse takotsubo cardiomyopathy. Patient was observed on the general floor for 3 days and then discharged home with outpatient follow up with cardiology planned. Patient reported gradual return of cardiopulmonary status to baseline over the next few months. As expected, a repeat echocardiogram done 3 months later showed return of cardiac function to normal.

\section{Discussion}

Takotsubo cardiomyopathy was first described in 1990 and has since been recognized as an important form of nonischemic cardiomyopathy. ${ }^{[1]}$ Recent advances have been made in understanding its pathophysiology. Both emotional and physical stresses have been identified as precipitants. Catecholamine surge seems to be the common denominator that directly leads to cardiomyopathy in the genetically predisposed. Postmenopausal females are especially at risk. It has been established that at critically high epinephrine con- centrations, beta-2 adrenergic receptor switches its coupling from the Gs protein to an inhibitory Gi protein. ${ }^{[2]}$ This results in negative inotropic effect. The apical myocardium has been found to have a higher ratio of beta- 2 to beta- 1 receptors, which explains the selective negative inotropic effects of catecholamine surge in the apex.

Subsequently, other variants of takstsubo cardiomyopathy have been identified. Reverse (inverse) takotsubo cardiomyopathy is a variant where the pattern of ventricular wall motion abnormalities is essentially opposite to that of takotsubo cardiomyopathy: there is apical sparing with akinesis of proximal ventricular walls. Various features of this lesser common variant have been compared to the apical variant. In one such analysis of 103 patients, the group with inverted takotsubo cardiomyopathy was younger (median age 54.5 vs $64.0 ; p=.006)$, and had a higher prevalence of triggering stress $(100 \%$ vs $77 \%$; $p=.018)$ when compared to mid or apical variants. ${ }^{[3]}$ In another study involving 60 patients, patients with inverted takotsubo cardiomyopathy had a mean age of 36 years versus 62 years in other subtypes. ${ }^{[4]}$ Our patient was 58-years old, and was thus an outlier when compared to these studies. Of note, our patient also had right ventricular dysfunction. Right ventricular dysfunction has been reported in a small fraction of patient with takotsubo cardiomyopathy and is associated with more severe impairment of left ventricular function. ${ }^{[5]}$

The clinical features and management of all variants of takotsubo cardiomyopathy are identical. Acute substernal chest pain is the most common presenting symptom, followed by dyspnea and syncope. Approximately $10 \%$ of the patients develop clinical features of shock. Our patient had an atypical presentation as she did not have any chest pain. She had no features of cardiogenic shock such as hypotension, encephalopathy, or cold and clammy extremities. However, her left ventricular output was severely reduced, which likely contributed to non-ischemic mesenteric ischemia. Hence, abdominal pain was the only initial presenting symptom. Patient was most likely hypovolemic on presentation because of diarrhea. This may be why pulmonary congestion was not obvious on presentation. However, when she was aggressively volume resuscitated, she subsequently developed acute pulmonary congestion and pulmonary edema. EKG changes including ST elevation, ST depression, and T wave inversion may be seen in these patients. Cardiac biomarkers are elevated in most patients. Coronary angiogram, if performed, shows normal coronary vessels or mild to moderate coronary atherosclerosis. Treatment is supportive and most patients have complete recovery.

Non-occlusive mesenteric ischemia (NOMI) is a form of 
acute intestinal ischemia that is characterized by bowel ischemia secondary to global mesenteric hypoperfusion. This occurs in the setting of low cardiac output states, as opposed to acute mesenteric arterial embolism or thrombosis. ${ }^{[6]}$ It is usually seen in critically ill patients who have severe cardiovascular derangements e.g. cardiogenic shock, septic shock etc. ${ }^{[7]}$ Although our patient didn't strictly meet clinical or hemodynamic criteria $\left(1.8 \mathrm{~L} / \mathrm{min} / \mathrm{m}^{2}\right)$ for cardiogenic shock, her cardiac index was severely reduced $\left(1.9 \mathrm{~L} / \mathrm{min} / \mathrm{m}^{2}\right)$. Splanchnic vasoconstriction associated with these low cardiac output states further reduces bowel perfusion. This effect is magnified in patients on vasopressor agents. Bowel regions most predisposed to NOMI are the watershed areas where collateral vessels bridge two arterial distributions. The splenic flexure lies at the junction of the blood supply of superior and inferior mesenteric arteries and is the most important watershed area. ${ }^{[6]}$

The clinical picture in patients with NOMI is often dominated by the primary inciting event. As noted before, our patient had an atypical presentation as abdominal complaint due to NOMI was her presenting complaint. It should be noted that the mortality of NOMI is high, and mortality rates in excess of $50 \%$ have been reported. ${ }^{[8]}$ Our patient had a more favorable outcome as given the reported rates. Abdominal pain

\section{REFERENCES}

[1] Sato H, Tateishi H, Uchida T. Takotsubo-type cardiomyopathy due to multivessel spasm. In: Kodama K, Haze K, Hon M, editors. Clinical Aspect of Myocardial Injury: From Ischemia to Heart Failure. Tokyo, Japan: Kagakuhyouronsha. 1990: 56-64. PMid:2239249

[2] Paur H, Wright PT, Sikkel MB, et al. High levels of circulating epinephrine trigger apical cardiodepression in a $\beta 2$-adrenergic receptor/Gi-dependent manner: A new model of Takotsubo cardiomyopathy. Circulation. 2012 Aug 7; 126(6): 697-706. PMid:22732314 https://doi.org/10.1161/CIRCULATIONAHA.112.111591

[3] Song BG, Chun WJ, Park YH, et al. The clinical characteristics, laboratory parameters, electrocardiographic, and echocardiographic findings of reverse or inverted takotsubo cardiomyopathy: Comparison with mid or apical variant. Clin Cardiol. 2011 Nov; 34(11): 693-9. PMid:22031226 https://doi .org/10.1002/clc. 20953

[4] Ramaraj R, Movahed MR. Reverse or inverted takotsubo cardiomyopathy (reverse left ventricular apical ballooning syndrome) presents at a younger age compared with the mid or apical variant and is is often mild to moderate as compared to the classic severe pain seen in patients with acute mesenteric arterial occlusion. Abdominal pain can be absent in $25 \%$ of the patients with NOMI. The diagnosis is made by radiological detection of colitis in watershed areas in a patient with low cardiac-output state. Treatment is mainly supportive. Aggressive hemodynamic monitoring and support is required. Complications such as transmural bowel infarction and perforation are rare and warrant urgent surgical intervention.

\section{Conclusion}

Reverse takotsubo cardiomyopathy is characterized by basal akinesis with apical sparing. Recognition of reverse takotsubo cardiomyopathy is important for diagnosis and prognostication of new-onset cardiomyopathy. Development of pulmonary congestion in low cardiac output states is dependent on the fluid status. Severe hypervolemia from aggressive fluid therapy can result in acute hypoxic respiratory failure due to hydrostatic pulmonary edema. NOMI can be caused by global mesenteric ischemia in the setting of low cardiac output states. Ischemia of the splenic flexure (watershed area) is a common manifestation of NOMI.

\section{CONFLicts OF INTEREST Disclosure}

The authors have declared no conflicts of interest. always associated with triggering stress. Congest Heart Fail. 2010 Nov-Dec; 16(6): 284-6. PMid:21091614 https ://doi .org/10.1 $111 / j .1751-7133.2010 .00188 . x$

[5] Haghi D, Athanasiadis A, Papavassiliu T, et al. Right ventricular involvement in Takotsubo cardiomyopathy. Eur Heart J. 2006 Oct; 27(20): 2433-9. PMid:17000628 https ://doi .org/10.1093/eu rheartj/ehl274

[6] Clair DG, Beach JM. Mesenteric Ischemia. N Engl J Med. 2016 Mar 10; 374(10): 959-68. PMid:26962730 https://doi.org/10.105 6/NEJMra1503884

[7] Cappell MS, Mahajan D, Kurupath V. Characterization of ischemic colitis associated with myocardial infarction: An analysis of 23 patients. Am J Med. 2006 Jun; 119(6): 527. e1-9.

[8] Trompeter M, Brazda T, Remy CT, et al. Non-occlusive mesenteric ischemia: Etiology, diagnosis, and interventional therapy. Eur Radiol. 2002 May; 12(5): 1179-87. PMid:11976865 https: //doi.org/10.1007/s00330-001-1220-2 